\title{
AVALIAÇÃO DAS APRENDIZAGENS: UM ESTUDO BIBLIOMÉTRICO
}

\author{
Eduardo Amadeu Dutra Moresi ${ }^{1}$, Isabel Pinho², Vilson Carlos Hartmann ${ }^{1}$, Mário de Oliveira \\ Braga Filho', Cláudia Pinho², António Pedro Costa ${ }^{2}$ \\ 1Universidade Católica de Brasília, Brasil. moresi@ucb.br; hartmann@ucb.br; braga@ucb.br \\ ²universidade de Aveiro, Portugal. isabelpinho@ua.pt; claudiapinho@ua.pt; apcosta@ua.pt
}

\begin{abstract}
Resumo. Para além de garantir o acesso universal à educação é necessário saber se os estudantes aprendem. Assim a avaliação das aprendizagens pode contribuir para uma educação de qualidade. A questão inicial de pesquisa, selecionada pela equipe de investigação, foi: o que é a Avaliação das Aprendizagens? Com vista a responder a esta questão, fez-se um estudo exploratório sobre este complexo tema, com o objetivo de obter um mapeamento da sua estrutura. Através de uma análise bibliométrica de dados recolhidos a partir da Scopus foi possível fazer uma análise exploratória de 3983 documentos, no período de 1971 a 2020. A partir dos metadados destes documentos fez-se análise da coocorrência das palavras-chave e da cocitação desses documentos. Os dados foram tratados com o apoio de vários softwares que nos permitiram obter resultados significativos, de modo a selecionarmos uma amostra de artigos relevantes. Esta amostra será objeto de uma futura análise de conteúdo. Para além dos resultados obtidos, o trabalho em equipe revelou-se uma mais-valia para o desenvolvimento da revisão da literatura onde cada um dos membros poderá contribuir com a sua experiência para a criação de conhecimento relevante sobre este tema. Esta síntese será fruto da sinergia do trabalho colaborativo de investigação/pesquisa interinstitucional e internacional.
\end{abstract}

Palavras-chave: Avaliação de Aprendizagens; Bibliometria; Metadados; Visualização; Avaliação Educacional.

\section{LEARNING ASSESSMENT: A BIBLIOMETRIC STUDY}

Abstract. In addition to ensuring universal access to education, it is necessary to know whether students learn. Thus, the learning assessment can contribute to quality education. The initial research question, selected by the research team, was: what is Learning Assessment? In order to answer this question, an exploratory study was carried on this complex topic, with the objective of obtaining a mapping of its structure. Through a bibliometric analysis of data collected from Scopus, it was possible to make an exploratory analysis of 3983 documents, from 1971 to 2020 . From the metadata of these documents, an analysis was made of the co-occurrence of the keywords and the co-citation of these documents. The data were analyzed with the support of various software that allowed us to obtain significant results, in order to select a sample of relevant articles. This sample will be the subject of a future content analysis. In addition to the results obtained, the teamwork proved to be an asset for the development of the literature review where each member can contribute with their expertise to create relevant knowledge on this topic. This synthesis will be the result of the synergy of the collaborative work of interinstitutional and international research.

Keywords: Learning Assessment; Bibliometric; Metadata; Visualization; Educational Evaluation.

\section{INTRODUÇÃO}

A história da avaliação de aprendizagens não é linear, processando-se, naturalmente, em espirais de conhecimentos adquiridos pelas sucessivas experiências de implementação. tema da avaliação das aprendizagens é complexo e insere-se na área da avaliação educacional. A sua evolução como tema de investigação/pesquisa e como prática tem evoluído com a contribuição de várias áreas do conhecimento. 
Para melhor compreender esta complexidade, torna-se necessário mapear a sua estrutura conceptual, de modo a obter uma visão integrativa das diversas dimensões temáticas estruturantes, bem como dos artigos e autores considerados relevantes com elevado impacto no desenvolvimento do tema.

A questão de pesquisa - o que é a Avaliação das Aprendizagens - é o ponto de partida para realizar este estudo exploratório. Trata-se de um estudo bibliométrico de um conjunto de artigos recuperados em uma base de dados de referência como é a Scopus. A escolha desta base de dados se baseia na sua abrangência geográfica global de publicação científica, que permite o acesso a excelente conteúdo, especialmente para as Ciências Sociais, bem como a disponibilização de metadados dos documentos que são úteis para serem explorados por diversos softwares de análise bibliométrica.

Assim, a partir de algumas publicações seminais, pode-se construir o protocolo ou ficha de pesquisa onde estão registradas as decisões tomadas para proceder à procura sistemática na base de dados, para ser respondida a questão de investigação/pesquisa proposta acima.

Para simplificar, pode-se indicar algumas publicações seminais sobre avaliação como sejam a de Michel Scriven (Scriven, 1967) que introduz o conceito de "avaliação formativa", apresentando ainda argumentos a favor e contra o recurso a tipos de avaliação formativa ou sumativa.

Outra publicação importante, nesta análise histórica, é o artigo de Benjamim Bloom (Bloom, 1968), onde se sustenta que a valorização da aprendizagem do estudante gera alterações na concepção do ato de ensinar, resultando daí um padrão de desempenho escolar semelhante à distribuição da curva normal. Bloom argumentou ainda que os professores deveriam diferenciar a instrução para melhor atender às necessidades individuais de aprendizagem dos estudantes, porque estes têm diferentes estilos e aptidões para aprender. Bloom e os seus colegas (Bloom et al., 1971) publicaram ainda um livro intitulado "Handbook on Formative and Summative Evaluation of Student Learning", direcionado para o professor fazer a avaliação na sala de aula.

A avaliação é um processo complexo, mas que pode ser apresentado de modo simplificado, para clarificar e harmonizar os conceitos, os objetivos e os critérios escolhidos. Earl e Katz (2006) fornecem uma estrutura para ajudar a pensar sobre os propósitos da avaliação, bem como para ajudar a criar e implementar as mudanças nas práticas de avaliação empregadas 
pelos professores, que sejam consistentes com a melhoria da aprendizagem para todos os estudantes. Trata-se de uma abordagem processual que fornece uma visão transversal sobre os principais temas estruturantes desta área de investigação e da sua aplicação.

Assim, considera-se que a avaliação parte de um conjunto de recursos que são processados para fornecer resultados. A avaliação pode ser assim ser considerada como uma sequência de etapas que incluem variadas tarefas (pensar, planejar e implementar). Os resultados obtidos devem ser compreendidos, usados de acordo com os diversos propósitos previamente definidos e utilizados por diferentes interessados. Esta abordagem transversal permite começar a construir o modelo conceitual que irá ser enriquecido com os resultados obtidos do presente estudo bibliométrico.

Nas duas seções seguintes serão apresentados a metodologia utilizada e os resultados obtidos. Nas conclusões serão indicadas as futuras fases a serem realizadas de modo a obter uma amostra de publicações relevantes que irão ser objeto de uma análise de conteúdo, que fará parte de uma revisão de literatura integrativa sobre a avaliação das aprendizagens (Pinho \& Leite, 2014; Pinho et al., 2014; Soares et al., 2019).

\section{METODOLOGIA}

Este trabalho tem como objetivo apresentar uma análise bibliométrica do tema Avaliação das Aprendizagens para responder à questão de pesquisa, explorando a análise de redes de coocorrência de palavras-chave e de cocitações de documentos. Para isso, foram utilizados métodos de pesquisa bibliométrica (Zupic \& Cater, 2014) e de análise de redes (Newman, 2009; Van Eck \& Waltman, 2014).

De maneira geral, a bibliometria é a aplicação de métodos matemáticos e estatísticos aos livros e outros meios de comunicação escrita (Pritchard, 1969), abrangendo as publicações em geral. Uma rede bibliométrica consiste em grafos com nós ou vértices (unidades de análise) e arestas (tipos de análises). Os nós podem ser, por exemplo, publicações, periódicos, pesquisadores, países, organizações ou palavras-chave. As arestas indicam relações entre pares de nós. Os tipos de relações mais comumente estudados empregam métodos bibliométricos compreendendo as de citação, de coocorrência de palavras-chave e de coautoria. No caso das relações de citação, uma distinção adicional pode ser feita entre as de citação direta, de cocitação e de acoplamento bibliográfico. 
Baseando-se em práticas metodológicas estabelecidas e na literatura sobre bibliometria, Zupic e Cater (2014) propuseram diretrizes de fluxo de trabalho recomendadas para a pesquisa de mapeamento científico utilizando os métodos bibliométricos. Eles não pretenderam apresentar um guia detalhado de instruções, mas uma visão geral do processo com as opções disponíveis aos pesquisadores (métodos, bancos de dados, software, etc.) e as decisões a serem tomadas em cada estágio da pesquisa.

A partir desse referencial de Zupic e Cater (2014), esta pesquisa seguiu as seguintes etapas: desenho da pesquisa com a definição do objetivo e a expressão de busca; escolha das unidades de análise - palavras-chave, documentos ou referências citadas; escolha do tipo de análise - redes de coocorrência de palavras-chave e de citações de documentos; seleção da base Scopus para a pesquisa bibliográfica e a recuperação de informações; escolha do software VOSviewer (Van Eck \& Waltman, 2019) para gerar as redes de coocorrência de palavras-chave e de cocitações de documentos; elaboração do arquivo de tesauros do VOSviewer para controle do vocabulário e normalização das referências bibliográficas; obtenção das redes de coocorrência de palavras-chave e de cocitações de documentos, com o controle do vocabulário e a normalização das referências bibliográficas; escolha do software Gephi (Bastian, Heymann, \& Jacomy, 2009) para o cálculo das métricas de análise de redes - grau médio, classes de modularidade e centralidade de autovetor; identificação dos termos mais relevantes e das referências com maiores centralidades de autovetor; visualização e interpretação dos resultados da rede de coocorrência de palavras-chave utilizando o software yEd.

Queremos reforçar que este estudo é exploratório e que os seus resultados são o ponto de partida para uma análise de conteúdo (Bardin, 2004) a realizar a uma amostra significativa de artigos. Esta amostra de artigos relevantes serão objeto de classificação e categorização. As categorias a considerar não só emergem deste estudo como das categorias prévias que constituíram algumas das palavras-chave usadas para construir as expressões de pesquisa na base de dados, que estão descriminadas na secção seguinte. 


\section{RESULTADOS DA PESQUISA}

O mapeamento científico com métodos bibliométricos é útil principalmente para ajudar os pesquisadores a explorar rapidamente a estrutura de uma área científica e introduzir rigor e transparência no processo de revisão da literatura. Cabe ressaltar que esses métodos não substituem a leitura extensiva e a síntese da literatura, mas antes podem fazer parte de uma fase exploratória para a seleção de uma amostra de literatura relevante, considerada como a que melhor pode contribuir para dar resposta a nossa questão de investigação/pesquisa. A bibliometria pode conectar de forma confiável publicações, autores ou periódicos; identificar substratos de pesquisa; e produzir mapas de pesquisas publicadas, mas cabe ao pesquisador possuir conhecimento sobre a área científica para interpretar as descobertas - que é a parte difícil.

A base Scopus possui alguns recursos para a análise bibliométrica, tais como o crescimento do tema em um período de tempo, os autores com maior frequência de documentos publicados, evolução dos quantitativos de artigos publicados em periódicos, distribuição por afiliação e países dos autores, além das áreas do conhecimento das publicações e os respectivos tipos de documentos.

Para Ball (2017), a ideia básica de uma pesquisa bibliométrica é quantificar a produção acadêmica de pessoas e instituições, permitindo que, em um segundo passo, conclusões qualitativas sejam extraídas destes dados. Já uma análise exploratória pode ser considerada o primeiro passo de qualquer trabalho científico pois proporciona informações sobre o tema que o pesquisador pretende abordar, auxiliando-o a definir seus objetivos, a formular suas hipóteses de trabalho e descobrir a melhor forma de desenvolver seu assunto. Pode ser feita a partir de documentos, bibliografias, visitas a páginas da web, etc (Ciribelli, 2003).

Nesse sentido, foi realizada uma pesquisa na base Scopus utilizando o termo education, que retornou 1.749.729 documentos publicados em periódicos e conferências, no período de 1860 a 2020. Em seguida, foi realizado um recorte com a expressão contendo as palavras-chave foco dessa pesquisa: "learning assessment" OR "learning evaluation" OR "classroom evaluation" OR "classroom assessment" OR "formative evaluation" OR "formative assessment" OR "summative evaluation" OR "summative assessment" OR "assessment of learning" OR "evaluation of learning" OR "evaluation for learning" OR "assessment for learning" OR "evaluation as learning" OR "assessment as learning". A pesquisa bibliográfica retornou 3.983 documentos, no período de 1.971 a 2020. A Figura 1 apresenta os percentuais 
por área temática. Observa-se que as áreas com maiores percentuais de documentos são Ciências Sociais com 54,9\%, Ciência da Computação com 8,5\% e Psicologia com 6,5\%.

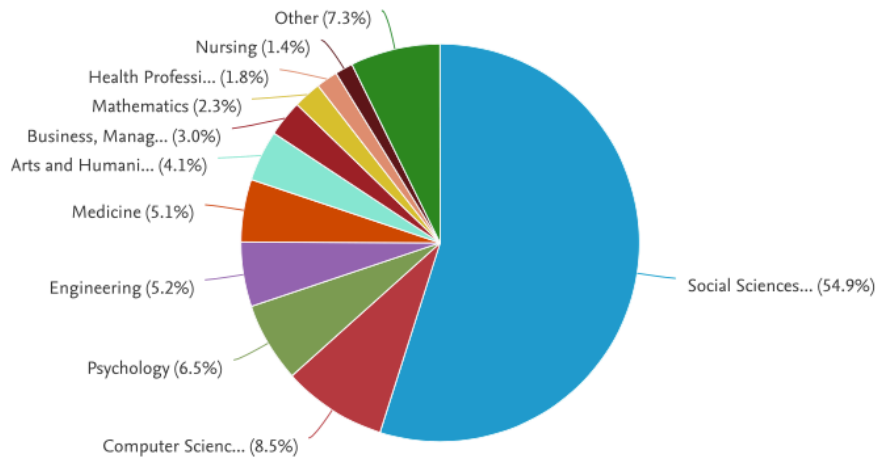

Figura 1. Percentual de documentos por área do conhecimento.

\subsection{Rede de coocorrência de palavras-chave}

As redes de coocorrências de palavras-chave são obtidas a partir da extração de termos do título e do resumo de uma publicação ou da lista de palavras-chave fornecidas pelo autor de uma publicação. Em alguns casos, as palavras-chave são restritas a uma única palavra, mas em outros também incluem termos que consistem em várias palavras, de acordo com o nível de aprofundamento a que a investigação/pesquisa se encontra. O número de coocorrências de duas palavras-chave corresponde à quantidade de publicações nas quais as duas palavras-chave ocorrem juntas no título, no resumo ou na lista de palavras-chave (Van Eck \& Waltman, 2014).

Assim, a análise de coocorrência de palavras-chave foi gerada utilizando o software VOSviewer (Van Eck \& Waltman, 2019). Os metadados foram lidos e selecionada a opção de coocorrência de palavras-chave do autor. Sem o controle do vocabulário, com um mínimo de 5 ocorrências para cada par de palavras-chave, resultou em uma rede de coocorrência com 410 termos, 12 comunidades e 4.310 arestas. Contudo, o VOSviewer oferece uma opção de criar um arquivo texto para realizar o controle do vocabulário - o tesauros, ou seja, a substituição e a exclusão de termos. Trata-se de uma interpretação qualitativa das palavraschave incluídas pelos autores nos metadados de cada documento. Com essa opção do controle do vocabulário, a rede resultante incluiu 216 termos, 14 clusters e 1. 326 arestas.

A Figura 2 apresenta a visualização do mapa de calor da rede de coocorrência de palavraschave. Algumas palavras-chave se destacam na rede: formative assessment, feedback, accountability, summative assessment, educational assessment, blended learning, self- 
assessment, learning outcomes, entre outras. Contudo, alguns termos que aparecem com baixa intensidade e em áreas mais periféricas da figura, podem representar oportunidades de pesquisa: creativity, pisa, assessment tools, student performance, assessment design, experiential learning, social justice, etc.

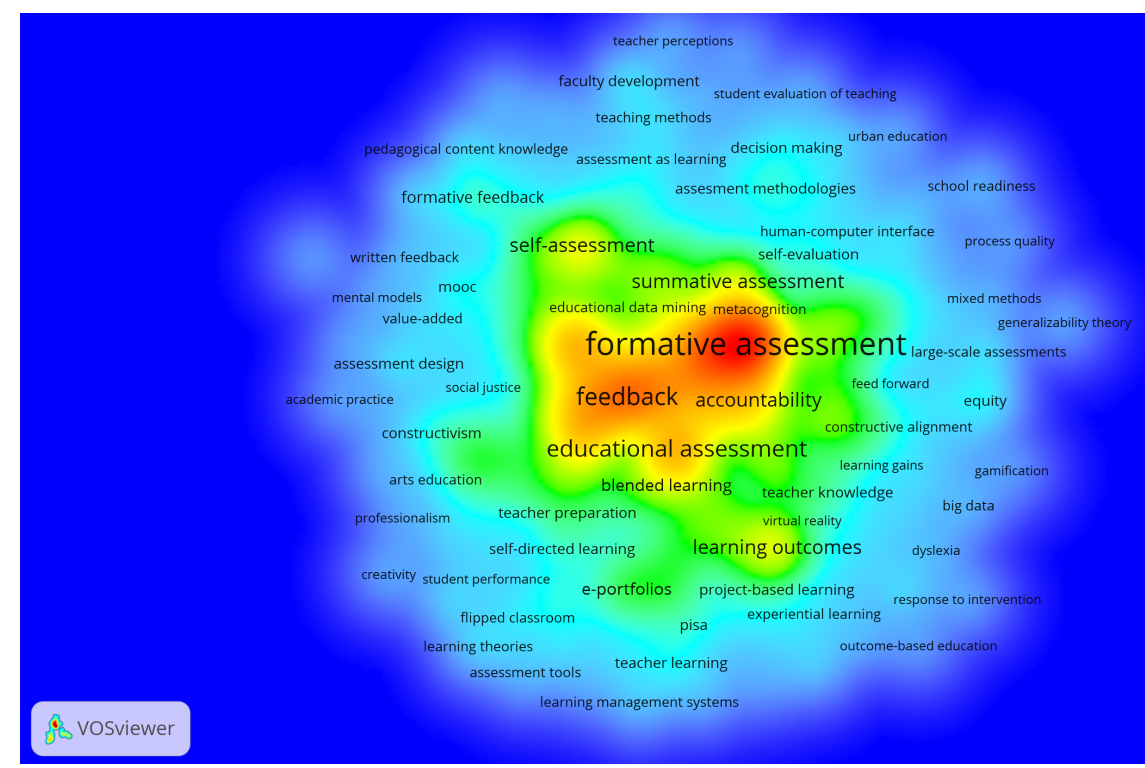

Figura 2. Visualização da rede de coocorrência de palavras-chave.

A rede foi exportada em formato GML para ser recuperada no Gephi (Bastian, Heymann, \& Jacomy, 2009), de modo a serem calculadas as métricas de redes: modularidade de classes e centralidades de grau e de autovetor. A centralidade de autovetor atribui relevância a um nó em função de sua relação com os demais vértices da rede. Se um nó está ligado a outros que se encontram em uma posição central na rede, o mesmo terá um valor alto de centralidade de autovetor. Já a centralidade de grau representa um importante aspecto da posição de um nó na estrutura de uma rede, além de atribuir a sua relevância em função do número de arestas que o mesmo estabelece com os demais vértices da rede (Newman, 2009).

A Tabela 1 apresenta os resultados para as 15 palavras-chave com valores mais elevados de centralidades de autovetor e de grau. A palavra-chave formative assessment é a que apresenta os maiores valores das centralidades calculadas.

Como o seu grau é igual a 118 e a palavra-chave seguinte possui 73 arestas, há uma grande variação nos valores da centralidade de autovetor. 
A lista de palavras-chave e de arestas foram exportadas em formato CSV e recuperadas em uma planilha Excel, para serem analisadas no yEd, que é um software livre para a edição de grafos. Esse tipo de representação permite visualizar o relacionamento entre as palavraschave e explicitar a estrutura de um campo de investigação/pesquisa.

A Figura 3 apresenta a estrutura conceitual a partir das palavras-chave: learning assessment, assessment methods, assessment as learning, assessment for learning. Cada caixa representa uma classe de modularidade, que foi calculada pelo Gephi. A denominação de classe foi atribuída a partir do termo com maior centralidade de autovetor. Observa-se como o tema central deste estudo - avaliação das aprendizagens - está relacionado direta e indiretamente às palavras-chave que representam a sua estrutura de pesquisa. Esta representação não é completa, pois depende da expressão de busca inicialmente utilizada para recuperar os documentos. Contudo, permite o aprofundamento do tema a partir da construção de novas expressões de busca com a combinação de palavras-chave e operadores lógicos.

Tabela 1. Palavras-chave com os valores mais elevados de centralidade de autovetor.

\begin{tabular}{lcc}
\hline \multicolumn{1}{c}{ Palavra-chave } & Grau & $\begin{array}{c}\text { Centralidade de } \\
\text { autovetor }\end{array}$ \\
\hline formative assessment & 118 & 1,0000 \\
teacher education & 73 & 0,6669 \\
educational assessment & 59 & 0,5803 \\
feedback & 58 & 0,5601 \\
assessment for learning & 53 & 0,5380 \\
peer assessment & 49 & 0,5039 \\
self-assessment & 37 & 0,4451 \\
e-learning & 45 & 0,4302 \\
classroom-based assessment & 40 & 0,4182 \\
summative assessment & 34 & 0,4007 \\
professional development & 38 & 0,3534 \\
pedagogy & 25 & 0,3378 \\
accountability & 37 & 0,3346 \\
assessment literacy & 26 & 0,3158 \\
student learning & 28 & 0,3140 \\
\hline
\end{tabular}

\subsection{Rede de cocitação de documentos}

Dois documentos são cocitados se houver um terceiro que os cite. Quanto maior o número de documentos em que duas publicações são citadas concomitantemente, mais forte será a relação de cocitação entre elas (Griffith et al., 1974). 
Um modelo bibliométrico de cocitação define áreas coerentes de problemas de pesquisa classificando e agrupando os trabalhos científicos atuais por meio de referências comuns a grupos de trabalhos muito citados ou cocitados.

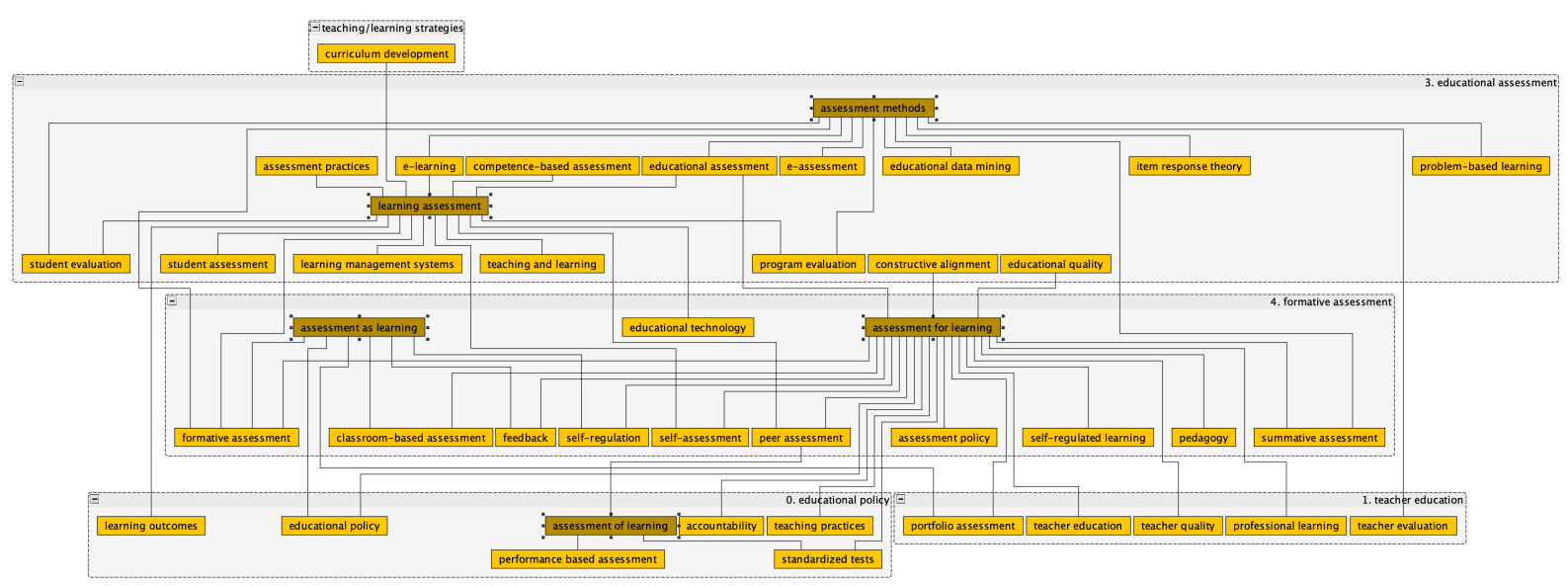

Figura 3. Visualização da estrutura do tema de pesquisa - learning assessment.

A unidade básica desse modelo é a comunidade de cocitação que é composta por dois componentes (Franklin \& Johnston, 1988): um conjunto de trabalhos citados e cocitados chamados de literatura de base; e um conjunto de artigos que os referenciam, denominada de literatura atual publicada do tema. Esse tipo de rede agrupa os trabalhos citados simultaneamente em um documento. Além disso, considera que a literatura de base representa os núcleos de teorias e métodos e os artigos citados descrevem as frentes de pesquisa em domínios temáticos no período investigado.

A rede de cocitação de documentos foi gerada utilizando o software VOSviewer (Van Eck, \& Waltman, 2019). Os metadados foram lidos e foi selecionada a opção de cocitação de documentos. O VOSviewer tem a opção, para este tipo de rede, de ser usado um arquivo de tesauros para normalizar as referências bibliográficas e tornar o resultado mais preciso. Sem o uso do arquivo de tesauros de referências bibliográficas, que elimina as referências duplicadas, com um mínimo de 10 cocitações para cada documento, obteve-se uma rede de cocitação com 203 nós e 3.884 arestas. Com a inclusão do tesauros de referências, a rede resultante passou a possuir 158 nós e 2.993 arestas.

O grafo foi exportado para o Gephi (Bastian, Heymann, \& Jacomy, 2009), onde foram calculadas as métricas de redes. A Tabela 2 apresenta os 10 documentos com as maiores centralidades de grau e de autovetor. $\mathrm{O}$ artigo com a maior centralidade é de Black e Wiliam (1998) e apresenta uma revisão de literatura sobre avaliação formativa em sala de aula. Eles 
incluiram uma análise mais detalhada e teórica da natureza do feedback, em que eles fornecem uma base para a discussão sobre o desenvolvimento de modelos teóricos para avaliação formativa e as perspetivas para o seu aprimoramento prático.

Tabela 2. Referências bibliográficas com as maiores centralidades de grau e de autovetor.

\begin{tabular}{|c|c|c|c|}
\hline Referência Bibliográfica & Foco & Grau & $\begin{array}{c}\text { Centralidade de } \\
\text { autovetor }\end{array}$ \\
\hline Black e Wiliam (1998) & avaliação formativa & 141 & 1,0000 \\
\hline Hattie e Timperley (2007) & feedback & 132 & 0,9625 \\
\hline Sadler (1989) & avaliação formativa & 124 & 0,9375 \\
\hline Black e Wiliam (2009) & avaliação formativa & 114 & 0,8539 \\
\hline Shute (2008) & feedback & 101 & 0,8202 \\
\hline Shepard (2000) & papel da avaliação & 109 & 0,8140 \\
\hline Wiliam (2011) & $\begin{array}{l}\text { avaliação formativa e de } \\
\text { aprendizaqem }\end{array}$ & 96 & 0,7912 \\
\hline Boud (2000) & avaliação sustentável & 89 & 0,7525 \\
\hline Bennett (2011) & avaliação formativa & 92 & 0,7437 \\
\hline $\begin{array}{l}\text { Nicol e Macfarlane-dick } \\
(2006)\end{array}$ & avaliação formativa e feedback & 84 & 0,7274 \\
\hline
\end{tabular}

Hattie e Timperley (2007) fornecem uma análise conceitual do feedback e analisam as evidências relacionadas ao seu impacto na aprendizagem e no desempenho, além de sugerir maneiras emprega-lo para aumentar sua eficácia em sala de aula. Sadler (1989) analisou a teoria da avaliação formativa particularmente em relação às habilidades que podem ser desenvolvidas para que os estudantes tenham experiências avaliativas autênticas e diretas. Black e Wiliam (2009) prosseguem na análise da avaliação formativa explorando teorias mais amplas da pedagogia e apresentam uma estrutura conceitual visando unificar um conjunto diversificado de práticas descritas como formativas.

Shute (2008) analisou o corpus de pesquisa sobre feedback, com foco no feedback formativo - definido como informações comunicadas ao estudante que se destinam a modificar seu pensamento ou comportamento para melhorar a aprendizagem. Shepard (2000) tratou da avaliação em sala, não para obter notas em provas, mas para ser usada como parte da aula visando apoiar e melhorar a aprendizagem. Wiliam (2011) discute as diferentes definições dos termos avaliação formativa e avaliação da aprendizagem e as inclui em uma definição mais ampla que se concentra na extensão em que as decisões instrucionais são apoiadas por evidências.

Boud (2000) analisa as práticas de avaliação em instituições de ensino superior com foco na avaliação sustentável, que abrange as habilidades necessárias para realizar as atividades que acompanham a aprendizagem ao longo da vida em contextos formais e informais. Bennett 
(2011) abordou seis questões inter-relacionadas à avaliação formativa, que dizem respeito à sua definição, às alegações comumente feitas sobre sua eficácia, à atenção limitada dada às considerações de domínio em sua conceituação, à sub-representação dos princípios de medição nessa conceituação, ao apoio do professor que exige avaliação formativa e ao impacto do sistema educacional maior. Ele conclui que o termo avaliação formativa ainda não representa um conjunto bem definido de artefactos ou práticas.

Nicol e Macfarlane-dick (2006) reinterpretaram a pesquisa sobre avaliação formativa e feedback para mostrar como esses processos podem ajudar os alunos a assumir o controle da sua própria aprendizagem, ou seja, a se tornarem aprendizes autorregulados.

Os artigos acima revelam os principais assuntos abordados nos 10 documentos cocitados com as maiores centralidades de autovetor. $O$ resultado é coerente com a análise de coocorrência de palavras-chave que apresenta a maior centralidade de autovetor para o termo avaliação formativa. O termo feedback também apresenta uma centralidade alta, ficando na quarta posição.

\section{CONCLUSÕES}

O mapeamento do tema através das variadas vertentes de visualização dos dados obtidos é uma fase importante para que se possa decidir sobre o foco a ser dado em uma futura revisão integrativa sobre a Avaliação das Aprendizagens. Trata-se de um tema que interessa a diversas áreas do conhecimento, mas que se vai construindo como área de investigação/pesquisa com teoria e prática estruturada. Autores e publicações relevantes são cocitados criando redes de conhecimento que consolidam este tema. Assim é possível identificar claramente tipos de avaliação de aprendizagem: Formativa (Bennett, 2011; Black \& Wiliam, 1998a; Black \& Wiliam, 1998b; Scriven, 1967); Sumativa (Dixson \& Worrell, 2016; Taras, 2005). Também é possível explorar as relações, como por exemplo a relação entre formativa e sumativa (Dolin et al., 2018) ou a relação entre a avaliação e as teorias de aprendizagem (Baird et al., 2017; Baird et al., 2014). Deste modo este estudo bibliométrico fornece resultados relevantes cuja interpretação e síntese passará a dar respostas a algumas questões específicas que começaram a emergir da discussão colaborativa sobre os mesmos resultados. Estas questões são elas próprias os alicerces da estrutura conceitual em construção. 
Assim, futuramente buscar-se-á dar respostas às seguintes questões sobre a Avaliação das Aprendizagens: Por quê avaliar? Avaliar o quê? Quais os métodos? Como garantir a qualidade? Como usar as informações? Como são usados os dados pelos diversos interessados? Que modelos nacionais de avaliação das aprendizagens estão implementados?

O grau de ensinamentos, com base na exploração e no aprofundamento da análise e interpretação dos dados obtidos, irá depender do conhecimento prévio de cada elemento da equipe de investigação.

Este trabalho a desenvolver será eficaz na medida da capacidade de trabalho de equipe, medida pelos resultados a obter dessa sinergia colaborativa. A discussão destes resultados nesta conferência por certo será outra contribuição relevante para o avanço deste trabalho. Reitera-se que a tomada de decisão sobre as direções futuras a tomar será sempre orientada pela questão de investigação/pesquisa principal e pelas questões específicas que possam emergir.

\section{REFERÊNCIAS}

Baird, J. A., Andrich, D., Hopfenbeck, T. N., \& Stobart, G. (2017). Assessment and learning: fields apart?. Assessment in Education-Principles Policy \& Practice, 24(3), 317-350.

Baird, J. A., Hopfenbeck, T. N., Newton, P., Stobart, G., \& Steen-Utheim, A. T. (2014). Assessment and learning: State of the field review. NO: Knowledge Center for Education, Oslo.

Ball, R. (2017). An introduction to bibliometrics - new development and trends. Cambridge: Chandos Publishing.

Bardin, L. (2004). Análise de Conteúdo (R. Luís \& A. Pinheiro, Trans.). Lisboa: Edições 70.

Bastian, M., Heymann, S., \& Jacomy, M. (2009). Gephi: An Open Source Software for Exploring and Manipulating Networks. In: Proceedings of the Third International ICWSM Conference, 361-362.

Bennett, R.E. (2011). Formative assessment: a critical review. Assessment in Education: Principles, Policy \& Practice, 18 (1), 5-25.

Black, P., \& Wiliam, D. (1998a). Assessment and classroom learning. Assessment in Education, 5 (1), 7-74.

Black, P., \& Wiliam, D. (1998b). Inside the Black Box Raising Standards Through Classroom Assessment. Phi Delta Kappan, 80(2), 139-148.

Black, P., \& Wiliam, D. (2009). Developing the theory of formative assessment. Educational Assessment, Evaluation and Accountability, 21 (1), 5-31.

Bloom, B. S. (1968). Learning for Mastery. Instruction and Curriculum. Evaluation comment, 1(2).

Bloom, B. S., Hastings, J. T., \& Madaus, G. F. (1971). Handbook on Formative and Summative Evaluation of Student Learning. New York: McGraw-Hill.

Boud, D. (2000). Sustainable assessment: rethinking assessment for the learning society. Studies in Continuing 
Education, $22(2), 151-167$.

Ciribelli, M. C. (2003). Como elaborar uma dissertação de mestrado através da pesquisa científica. Rio de Janeiro: 7 Letras.

Dixson, D. D., \& Worrell, F. C. (2016). Formative and Summative Assessment in the Classroom. Theory Into Practice, 55(2), 153-159.

Dolin, J., Black, P., Harlen, W., \& Tiberghien, A. (2018). Exploring Relations Between Formative and Summative Assessment. In J. Dolin \& R. Evans (Eds.), Transforming Assessment: Through an Interplay Between Practice, Research and Policy (pp. 53-80). Cham: Springer International Publishing.

Earl, L., \& Katz, S. (2006). Rethinking classroom assessment with purpose in mind. Winnipeg, Manitoba, Canada: Manitoba.

Franklin, J. J., \& Johnston, R. (1988). Co-citation bibliometric modelling for S\&T and R\&D management. In Van Raan, A. F. J. (Ed.). Handbook of Quantitative Studies of Science and Technology. Amsterdam: North Holland.

Griffith, B. C., Small, H., Stonehill, J. A., Dey, S. (1974). The structure of scientific literatures II: Toward a macroand microstructure for science. Science Studies, 4(4), 339-365.

Hattie, J., \& Timperley, H. (2007). The power of feedback. Review of Educational Research, 77 (1), 81-112.

Newman, M. (2009). Networks: an introduction. Oxford: Oxford University Press.

Nicol, D. J., \& Macfarlane-dick, D. (2006). Formative assessment and self-regulated learning: a model and seven principles of good feedback practice. Studies in Higher Education, 31, 199-218.

Pinho, I., \& Leite, D. (2014). Doing a literature review using content analysis - Research Networks Review. Poster CIAIQ 2014- Congresso Ibero-Americano em Investigação Qualitativa, Badajoz, Espanha, 4 - 16 de Julho.

Pinho, I., Rodrigues, L., Neri de Souza, F., \& Lopes, G. (2014). Determinantes na Adoção e Recomendação de Software de Investigação Qualitativa: Estudo Exploratório. Internet Latente Corpus Journal, 4(2), 91-102.

Pritchard, A. (1969). Statistical bibliography or bibliometrics? Journal of Documentation, 25 (4), 348-349.

Sadler, D.R. (1989). Formative assessment and the design of instructional systems. Instructional Science, 18 (2), 119-144.

Scriven, M. (1967). The methodology of evaluation. In R. G. Tyler \& M. Scriven (Eds.), Perspectives on Curriculum Evaluation (pp. 39-83). Chicago: Rand Mcnally.

Shepard, L.A. (2000). The role of assessment in a learning culture. Educational Researcher, 29 (7), 4-14.

Shute, V.J. (2008). Focus on formative feedback. Review of Educational Research, 78 (1), 153-189.

Soares, C. B., Fornari, L., Pinho, I., \& Costa, A. P. (2019). Revisão da Literatura com Apoio de Software Contribuição da Pesquisa Qualitativa. Portugal: Ludomedia.

Taras, M. (2005). Assessment - Summative and formative - Some theoretical reflections. British Journal of Educational Studies, 53(4), 466-478.

Van Eck, N. J., \& Waltman, L. (2019). VOSviewer manual. Leiden: Universiteit Leiden.

Van Eck, N. J., \& Waltman, L. (2014). Visualizing bibliometric networks. In: Ding, Y., Rousseau, R., \& Wolfram, D. (Eds.). Measuring scholarly impact: methods and practice. New York: Springer.

Wiliam, D. (2011). What is assessment for learning? Studies in Educational Evaluation, 37 (1), 3-14.

Zupic, I., \& Cater, T. (2014). Bibliometric methods in management organization. Organizational Research Methods, $18(3), 429-472$. 